\title{
Effects of Metformin on Colorectal Carcinogenesis
}

\author{
Paola Sena ${ }^{1}$, Monica Pedroni ${ }^{2}$ and Luca Roncucci*2 \\ ${ }^{1}$ Department of Biomedical, Italy \\ ${ }^{2}$ Department of Medical and Surgical Sciences, Italy \\ *Corresponding author: Luca Roncucci, Department of Medical and Surgical Sciences, Italy
}

\begin{abstract}
ARTICLE INFO
Received: 慧 February 05, 2019

Published: 幽 February 14, 2019

Citation: Paola Sena, Monica Pedroni, Luca Roncucci. Effects of Metformin on Colorectal Carcinogenesis. Biomed J Sci \& Tech Res 14(4)-2019. BJSTR. MS.ID.002574.

ABSTRACT

Metformin is widely used as an antidiabetic drug in patients with type II diabetes mellitus. In the last years several studies have tested the role of this substance in cancer treatment and prevention, relying on some interesting biological and functional properties of the molecule. Particular attention has been paid to colorectal carcinogenesis. In vitro studies, and intervention studies in animal models, have shown positive effects on biological and surrogate markers of colorectal cancer. In humans, results are still conflicting and inconsistent, however evidence from epidemiological and intervention studies suggests that metformin may reduce the risk of colorectal adenomas, thus exerting a possible chemopreventive effect on colorectal cancer development.
\end{abstract}

Keywords: Metformin; Colorectal Carcinogenesis; Apoptosis; Autophagy; Inflammation; Aberrant Crypt Foci; Colorectal Adenoma
Abbreviations: DMII: Diabetes Mellitus Type II; AMPK: AMP-Activated Protein Kinase; MTOR : Mechanistic Target of Rapamycin; ACF: Aberrant Crypt Foci

\section{Introduction}

Metformin (N,N-Dimethylimido-di-carbonimidic diamide) is a biguanidinic derivative used for the cure of diabetes mellitus type II (DMII). It was described by Werner and Bell in 1922. Its hypoglicemic effect was discovered in 1950, but only in 1957 J. Sterne tested it in diabetic patients. Metformin was introduced in clinical practice in Canada in 1970. In recent years, evidence has emerged that, besides the well-known properties of metformin suitable for its use as first-line therapy of DMII, it has some positive effects on key pathways related to cancer, and even to aging. In this review we will discuss in brief the evidence, coming from laboratory, experimental, epidemiological, and a few clinical studies, which suggests a possible use of metformin as chemopreventive drug in colorectal carcinogenesis, at least in selected groups of subjects at increased risk of large bowel malignancy, as those with colorectal adenomas.

\section{Effects of Metformin on Carbohydrate Metabolism}

The effects of metformin on glucose metabolism are mediated by the activation of the AMP-activated protein kinase (AMPK) in response to metabolic stress, which alters the ratio AMP/ATP, through increased consumption or reduced production of ATP, as following hypoxia, glucose deprivation, or inhibition of mitochondrial oxidative phsphorylation. AMPK drives cells from an anabolic to a catabolic state [1]. Metformin in type II diabetic patients inhibits hepatic gluconeogenesis and glycogenolysis, reduces insulin resistance in target tissues, and increases the uptake of glucose in peripheral tissues, especially in the skeletal muscles [2]. The main side effects of metformin are: nausea and vomiting, diarrhoea or constipation, abdominal discomfort, loss of appetite, usually not severe and revertible.

\section{Effects of Metformin on Cellular Functions and Metabolism}

In order to find possible explanations for the potential anticancer action of metformin, we should evaluate the effects of metformin on cell functions linked to cancer cell growth and survival advantage. The mechanistic target of rapamycin (mTOR), a serine/ threonine kinase, and the phosphatidilinositol-3-kinase (PI3K)/ Akt signalling pathways are strictly connected and involved in the control of cell growth and survival [3]. They integrate growth factor signals with cellular nutrient and energy balance. In this regard, 
the introduction of inhibitors and modulators (sirolimus, temsirolimus, everolimus, and second generation mTORC1/mTORC2 dual inhibitors) of the mTOR pathway is attractive and challenging, and they are actively studied also in colorectal cancer [4-7]. It has been hypothesized that metformin may inhibit the mTOR signalling via activation of the AMP-activated protein kinase (AMPK)-dependent and independent pathways [8]. Metformin has pro-apoptotic effects. It has been demonstrated on cell cultures at concentrations similar to doses used for diabetic patients $[9,10]$. This effect seems mediated by upregulation of bcl2-associated X protein [11]. Autophagy, a process triggered in cells in conditions of energy deprivation, is enhanced in cancer cells by metformin [12]. Furthermore, metformin can inhibit epithelial-mesenchimal transition, cell migration and metastasis in colon cancer cell cultures [13].

Metformin has also anti-inflammatory effects, through inhibition of NF- $\kappa$ B signalling in atherosclerosis animal models and in cell cultures [14,15]. Moreover, growing evidence suggests that metabolic syndrome, or overweight and obesity, are risk factor for several cancers including colorectal $[16,17]$ probably through increased production of inflammatory-related cytokines by the visceral adipose tissue. Interestingly, metformin seems to induce weight loss in obese people without diabetes [18].

\section{Effects of Metformin on Surrogate Biomarkers of Colorectal Cancer}

All the effects of metformin on various cellular functions, pathways, and metabolism may be extremely useful for colorectal cancer prevention, besides for cancer treatment. When considering colorectal cancer prevention, it is mandatory to evaluate the effects of lifestyle or chemopreventive strategies on precursor lesions of colorectal cancer. Indeed, metformin has been tested on earlier and surrogate markers of colorectal cancer risk, i.e., colorectal epithelial cell proliferation, aberrant crypt foci (ACF) and adenomas. ACF are microscopic lesions that develop on the surface of colorectal mucosa after carcinogen treatment in animal models [19] and in humans after exposure to environmental carcinogens, mainly of dietary origin [20]. Metformin has been shown to reduce colorectal epithelial cell proliferation, the number of ACF in Azoxymethane (AOM)-initiated mice [21] activating the AMPK pathway, and the number of intestinal polyps in APC Min/+ mice [22]. Metformin was also effective in reducing the inflammatory environment of the intestine, and oxidative stress parameters [23]. In humans, evidence is weaker, and results controversial, but the effects on epithelial cell proliferation and ACF seem similar [24]. Different doses of metformin $(250,500$, and $1500 \mathrm{mg} /$ die $)$ were tested in patients with impaired glucose tolerance, undergoing colonoscopy. The mean number of ACF per patients decreased, at 3 and 6 months of treatment, only in the 500 and $1500 \mathrm{mg}$ groups [25]. So far, only one randomized, placebo-controlled trial tested low dose metformin as chemopreventive agent in non-diabetic subjects after polypectomy at index colonoscopy [26]. The administration of metformin was safe, and after 1 year of treatment, patients taking metformin had a reduced prevalence and number of adenomas. Recent metanalyses and retrospective studies showed not conclusive results: as a whole, they seem to suggest that patients taking metformin have a reduced risk of colorectal adenomas [27-30] and one study found that metformin use was associated with reduced risk only for advanced adenomas [31].

\section{Conclusion}

In conclusion, recent evidence, based on pharmacological, biological and functional studies, along with a few epidemiological and clinical studies, though not conclusive, seems to suggest that metformin may have a beneficial effect on colorectal carcinogenesis. However, further clinical trials are needed in order to validate these preliminary results. Metformin may be considered as a promising chemopreventive agent in patients a high risk of developing colorectal cancer.

\section{References}

1. Zhou G, Myers R, Li Y, Chen Y, Shen X, et al. (2001) Role of AMP-activated protein kinase in mechanism of metformin action. J Clin Invest 108(8): 1167-1174.

2. Dunn CJ, Peters DH (1995) Metformin. A review of its pharmacological properties and therapeutic use in non-insulin-dependent diabetes mellitus. Drugs 49(5): 721-749.

3. Porta C, Paglino C, Mosca A (2014) Targeting PI3K/Akt/mTOR signaling in cancer. Front Oncol 4: 64 .

4. Silvestris N, Tommasi S, Petriella D, Santini D, Fistola E, et al. (2009) The dark side of the moon: the PI3K/PTEN/AKT pathway in colorectal carcinoma. Oncology 77(1): 57-68.

5. Wang XW, Zhang YJ (2015) Targeting mTOR network in colorectal cancer therapy. World J Gastroenterol pp. 4178-4188.

6. Francipane MG, Lagasse E (2014) mTOR pathway in colorectal cancer: an update. Oncotarget 5(1): 49-66.

7. Wang HW, Yang SH, Huang GD, Lin JK, Chen WS, et al. (2014) Temsirolimus enhances the efficacy of cetuximab in colon cancer through a CIP2Adependent mechanism. J Cancer Res Clin Oncol 140(4): 561-571.

8. Yue W, Yang CS, Dipaola RS, Tan XL (2014) Repurposing of metformin and aspirin by targeting AMPK-mTOR and inflammation for pancreatic cancer prevention and treatment. Cancer Prev Res (Phila) 7(4): 388-397.

9. Habibollahi P, Van Den Berg NS, Kuruppu D, Loda M, Mahmood U (2013) Metformin--an adjunct antineoplastic therapy--divergently modulates tumor metabolism and proliferation, interfering with early response prediction by 18F-FDG PET imaging. J Nucl Med 54(2): 252-258.

10. Sena P, Mancini S, Benincasa M, Mariani F, Palumbo C, et al. (2018) Metformin Induces Apoptosis and Alters Cellular Responses to Oxidative Stress in Ht29 Colon Cancer Cells: Preliminary Findings. Int J Mol Sci 19(5).

11. Fang Z, Xu X, Zhou Z, Xu Z, Liu Z (2014) Effect of metformin on apoptosis, cell cycle arrest migration and invasion of A498 cells. Mol Med Rep 9(6): 2251-2256.

12. Buzzai M, Jones RG, Amaravadi RK, Lum JJ, De Berardinis RJ, et al. (2007) Systemic treatment with the antidiabetic drug metformin selectively impairs p53-deficient tumor cell growth. Cancer Res 67(14): 6745-6752.

13. Kang S, Kim BR, Kang MH, Kim DY, Lee DH, et al. (2018) Anti-metastatic effect of metformin via repression of interleukin 6-induced epithelialmesenchymal transition in human colon cancer cells. Plos One 13(10).

14. Li SN, Wang X, Zeng QT, Feng YB, Cheng X, et al. (2009) Metformin inhibits nuclear factor kappaB activation and decreases serum high-sensitivity 
C-reactive protein level in experimental atherogenesis of rabbits. Heart Vessels 24(6): 446-453.

15. Tan BK, Adya R, Chen J, Farhatullah S, Heutling D, et al. (2009) Metformin decreases angiogenesis via NF-kappaB and Erk1/2/Erk5 pathways by increasing the antiangiogenic thrombospondin-1. Cardiovasc Res 83(3): 566-574.

16. Gathirua Mwangi WG, Song Y, Monahan PO, Champion VL, Zollinger TW (2018) Associations of metabolic syndrome and C-reactive protein with mortality from total cancer, obesity-linked cancers and breast cancer among women in NHANES III. Int J Cancer 143(3): 535-342.

17. Kabat GC, Kim MY, Stefanick M, Ho GYF, Lane DS, et al. (2018) Metabolic obesity phenotypes and risk of colorectal cancer in postmenopausal women. Int J Cancer 143(3): 543-551.

18. Hui F, Zhang Y, Ren T, Li X, Zhao M, Zhao Q (2018) Role of metformin in overweight and obese people without diabetes: a systematic review and network meta-analysis. Eur J Clin Pharmacol.

19. Bird RP (1987) Observation and quantification of aberrant crypts in the murine colon treated with a colon carcinogen: preliminary findings. Cancer Lett 37(2): 147-151.

20. Roncucci L, Stamp D, Medline A, Cullen JB, Bruce WR (1991) Identification and quantification of aberrant crypt foci and microadenomas in the human colon. Hum Pathol 22(3): 287-294.

21. Hosono K, Endo H, Takahashi H, Sugiyama M, Uchiyama T, et al. (2010) Metformin suppresses azoxymethane-induced colorectal aberrant crypt foci by activating AMP-activated protein kinase. Mol Carcinog 49(7): 662-671.

22. Tomimoto A, Endo H, Sugiyama M, Fujisawa T, Hosono K, et al. (2008) Metformin suppresses intestinal polyp growth in ApcMin/+ mice. Cancer Sci 99(11): 2136-2141.

23. Bordini HP, Kremer JL, Fagundes TR, Melo GP, Conchon Costa I, et al. (2017) Protective effect of metformin in an aberrant crypt foci mode

\section{ISSN: 2574-1241}

DOI: 10.26717.BJSTR.2019.14.002574

Luca Roncucci. Biomed J Sci \& Tech Res

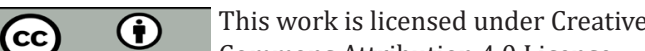

Submission Link: https://biomedres.us/submit-manuscript.php induced by 1,2-dimethylhydrazine: Modulation of oxidative stress and inflammatory process. Mol Carcinog 56(3): 913-922.

24. Hosono K, Endo H, Takahashi H, Sugiyama M, Sakai E, et al. (2010) Metformin suppresses colorectal aberrant crypt foci in a short-term clinical trial. Cancer Prev Res (Phila) 3(9): 1077-1083.

25. Zhao X, Li Y, Chen M, Chen Y, Dai Y, et al. (2015) Effects of different doses of metformin treatment for 6 months on aberrant crypt foci in Chinese patients with impaired glucose tolerance. Eur J Cancer Prev 24(1): 27 36.

26. Higurashi T, Hosono K, Takahashi H, Komiya Y, Umezawa S, et al. (2016) Metformin for chemoprevention of metachronous colorectal adenoma or polyps in post-polypectomy patients without diabetes: a multicentre double-blind, placebo-controlled, randomised phase 3 trial. Lancet Oncol 17(4): 475-483.

27. Hou YC, Hu Q, Huang J, Fang JY, Xiong H (2017) Metformin therapy and the risk of colorectal adenoma in patients with type 2 diabetes: A metaanalysis. Oncotarget 8(5): 8843-8853.

28. Liu F, Yan L, Wang Z, Lu Y, Chu Y, et al. (2017) Metformin therapy and risk of colorectal adenomas and colorectal cancer in type 2 diabetes mellitus patients: A systematic review and meta-analysis. Oncotarget 8(9): 16017-16026.

29. Han MS, Lee HJ, Park SJ, Hong SP, Cheon JH, et al. (2017) The effect of metformin on the recurrence of colorectal adenoma in diabetic patients with previous colorectal adenoma. Int J Colorectal Dis 32(8): 1223-1236.

30. Jung YS, Park CH, Eun CS, Park DI, Han DS (2017) Metformin use and the risk of colorectal adenoma: A systematic review and meta-analysis. J Gastroenterol Hepatol 32(5): 957-965.

31. Mansourian M, Karimi R, Vaseghi G (2018) Different effects of metformin and insulin on primary and secondary chemoprevention of colorectal adenoma in diabetes type 2: Traditional and Bayesian meta-analysis. EXCLI J 17: 45-56.

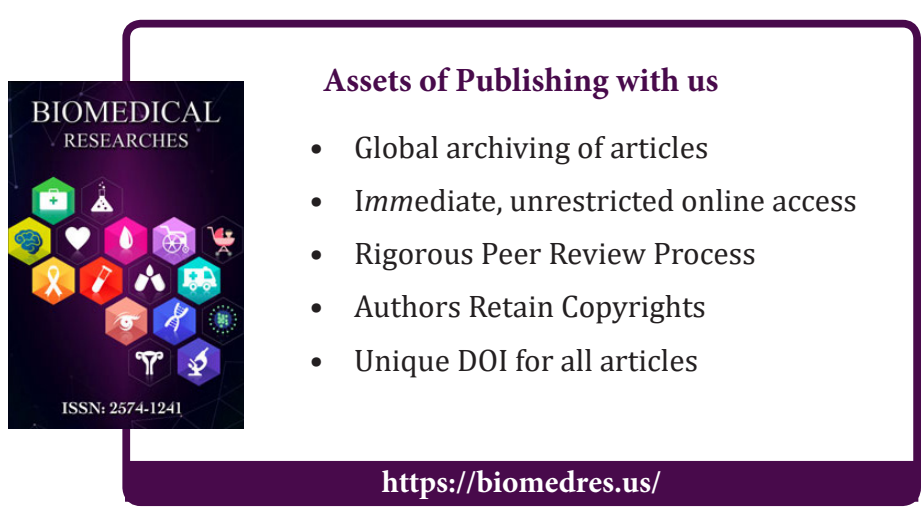

